This item was submitted to Loughborough's Research Repository by the author.

Items in Figshare are protected by copyright, with all rights reserved, unless otherwise indicated.

\title{
A biomechanical analysis of common lunge tasks in badminton
}

PLEASE CITE THE PUBLISHED VERSION

http://dx.doi.org/10.1080/02640410903428533

\section{PUBLISHER}

(C) Routledge (Taylor \& Francis Group)

VERSION

AM (Accepted Manuscript)

LICENCE

CC BY-NC-ND 4.0

\section{REPOSITORY RECORD}

Kuntze, Gregor, Neil J. Mansfield, and William Sellers. 2019. "A Biomechanical Analysis of Common Lunge Tasks in Badminton”. figshare. https://hdl.handle.net/2134/6009. 
This item was submitted to Loughborough's Institutional Repository (https://dspace.lboro.ac.uk/) by the author and is made available under the following Creative Commons Licence conditions.

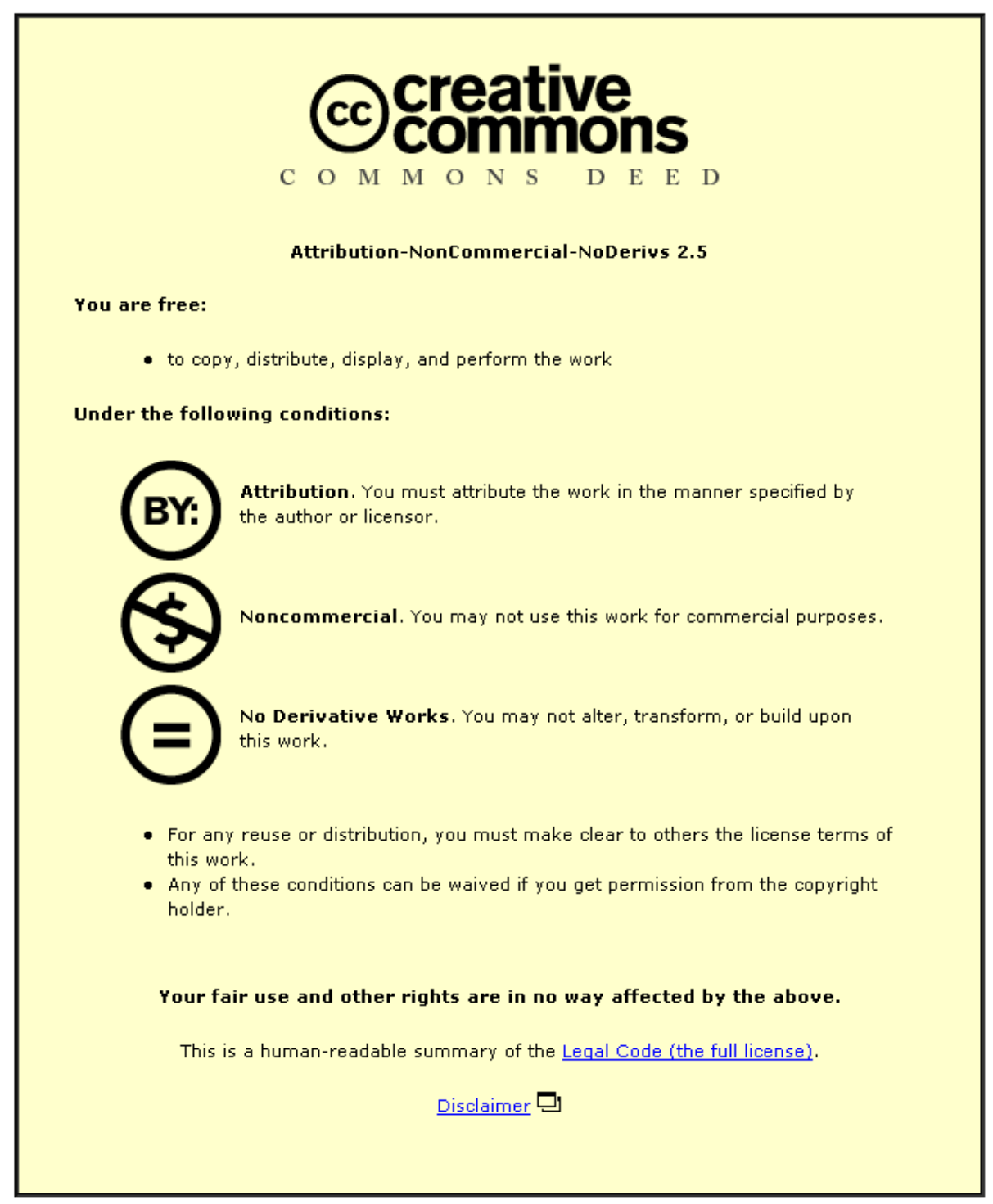

For the full text of this licence, please go to: http://creativecommons.org/licenses/by-nc-nd/2.5/ 


\title{
A biomechanical analysis of common lunge tasks in badminton
}

\author{
Authors: Gregor Kuntze a; Neil Mansfield ${ }^{\text {b; William Sellers }}{ }^{\text {c }}$ \\ Affiliations: ${ }^{a}$ Cardiff School of Sport, University of Wales Institute, Cardiff \\ ${ }^{\mathrm{b}}$ Department of Human Sciences, Loughborough University, Loughborough \\ ${ }^{\mathrm{c}}$ Faculty of Life Sciences, University of Manchester, Manchester, UK
}

DOI: $10.1080 / 02640410903428533$

Publication Frequency: 14 issues per year

Published in: $\vee$ Journal of Sports Sciences, Volume 28, Issue 2 January 2010 , pages 183 191

First Published on: 05 January 2010

\section{Abstract}

The lunge is regularly used in badminton and is recognized for the high physical demands it places on the lower limbs. Despite its common occurrence, little information is available on the biomechanics of lunging in the singles game. A video-based pilot study confirmed the

relatively high frequency of lunging, $\sim 15 \%$ of all movements, in competitive singles games.

The biomechanics and performance characteristics of three badminton-specific lunge tasks (kick, step-in, and hop lunge) were investigated in the laboratory with nine experienced male badminton players. Ground reaction forces and kinematic data were collected and lower limb joint kinetics calculated using an inverse dynamics approach. The step-in lunge was characterized by significantly lower mean horizontal reaction force at drive-off and lower mean peak hip joint power than the kick lunge. The hop lunge resulted in significantly larger mean reaction forces during loading and drive-off phases, as well as significantly larger mean peak ankle joint moments and knee and ankle joint powers than the kick or step-in lunges. These findings indicate that, within the setting of this investigation, the step-in lunge may be beneficial for reducing the muscular demands of lunge recovery and that the hop lunge allows for higher positive power output, thereby presenting an efficient lunging method.

Keywords: Joint moments; joint powers; lunge performance; ground reaction force

\section{Introduction}

Lunging forms an integral part of the movement repertoire of competitive athletes participating in sports such as badminton, squash, and fencing (Cronin, McNair, \& Marshall, 2003). In badminton, the lunge task allows the player to rapidly stop the progression of the body, form a secure base from which to play the necessary shot, and move back into the court to prepare for the next shot. The task, therefore, essentially consists of a weight acceptance (braking) and recovery (accelerating) phase and forms an integral part of the start-stoprecover cycle (Badminton Association of England, 2005). 
Considering the apparent importance of the lunge, particularly to sports such as badminton, it is surprising to find relatively little information on the kinematics and kinetics of this advanced movement skill in the literature. Lees and Hurley (1994) recorded the forces during a typical badminton lunge for a number of experienced and inexperienced participants, and identified modifications of movement technique to prevent excessive force exposure. A number of researchers have investigated the kinematics and kinetics of the fencing lunge (Adrian \& Klinger 1976; Gebhardt, 1981). Cronin et al. (2003) determined the strength qualities that act as important predictors to lunge performance, whereas others investigated lunging as a rehabilitation exercise (Heijne et al., 2004; Stuart, Meglan, Lutz, Growney, \& An, 1996).

Furthermore, Thijs and colleagues (Thijs, van Tiggelen, Willems, De Clercq, \& Witvrouw, 2007) assessed muscular strength parameters at the hip for a functional lunge movement in tennis. However, we are unaware of any studies that investigated different lunge tasks, including the recovery phase, in badminton. Therefore, there is a lack of objective data on performance criteria and joint kinetics during the lunge tasks, which may provide essential insight into the requirements of different lunge methods and may allow for task improvement by optimizing the movement processes and reducing joint loading and muscular demands.

The first aim of this research was to assess the frequency of the lunge to competitive singles badminton in a pilot study, using a notational analysis approach. Based on the findings of this pilot, a biomechanical investigation was used to assess differences in mechanical loading of the joints and performance differences between three badminton-specific lunge tasks. A further aim was to establish whether adoption of a novel lunge technique would result in enhanced task performance. A quick and mechanically efficient method of recovery may benefit the athlete by reducing the muscular demands of this important movement task, reduce muscular stress, and increase the time available to react to the following shot by reducing the total time of the lunge task.

\section{Methods}

\section{Pilot study}

A video-based notational analysis of men's and women's, national and international competitive singles games was performed to assess lunge frequency (see Table I for participant and video information). Video analysis was performed using frame-by-frame analysis. All movements performed by a player in a rally during the sampled period of time were counted and classed as belonging to one of six movement categories: running, sidestepping, crossover-stepping, lunging, jumping, and scrambling (containing all other unclassifiable movements).

Table I. Summary of participant and video information Games analysed

Number of
players $\begin{gathered}\begin{array}{c}\text { Mean } \\ \text { sampling } \\ \text { duration (s) }\end{array}\end{gathered}$


BUSA $=$ British Universities Sports Association.

Men, $2 \times$ World Cup semi-final, $2 \times$ World Cup

international quarter-final, $1 \times$ Thomas Cup Final,

Women, international

Men, national

$3 \times$ BUSA finals, $3 \times$ Satellite

Tournament

Women, national

$5 \times$ BUSA finals

8

58951

A lunge frequency of $\sim 15 \%$ of movements performed during competitive singles rallies was

identified, with a higher frequency of lunges at the international than the national standard $(17.86 \pm 4.83 \%$ and $14.29 \pm 4.51 \%$ respectively). These data identify frequency of use only and do not attempt to identify direction or magnitude of movement. Nevertheless, it appears that lunging forms an important function in the competitive singles game, with an increased application at the higher standard of competition.

While no distinction between lunge methods were made for frequency assessments, two distinct techniques appeared to be used in-game, referred to here as the kick and step-in lunges (see Figure 1 for a more detailed description). In addition, a third method, referred to here as the hop lunge, has been advocated recently. This method incorporates a hop during the weight acceptance phase, prior to lunge recovery (see Figure 1).

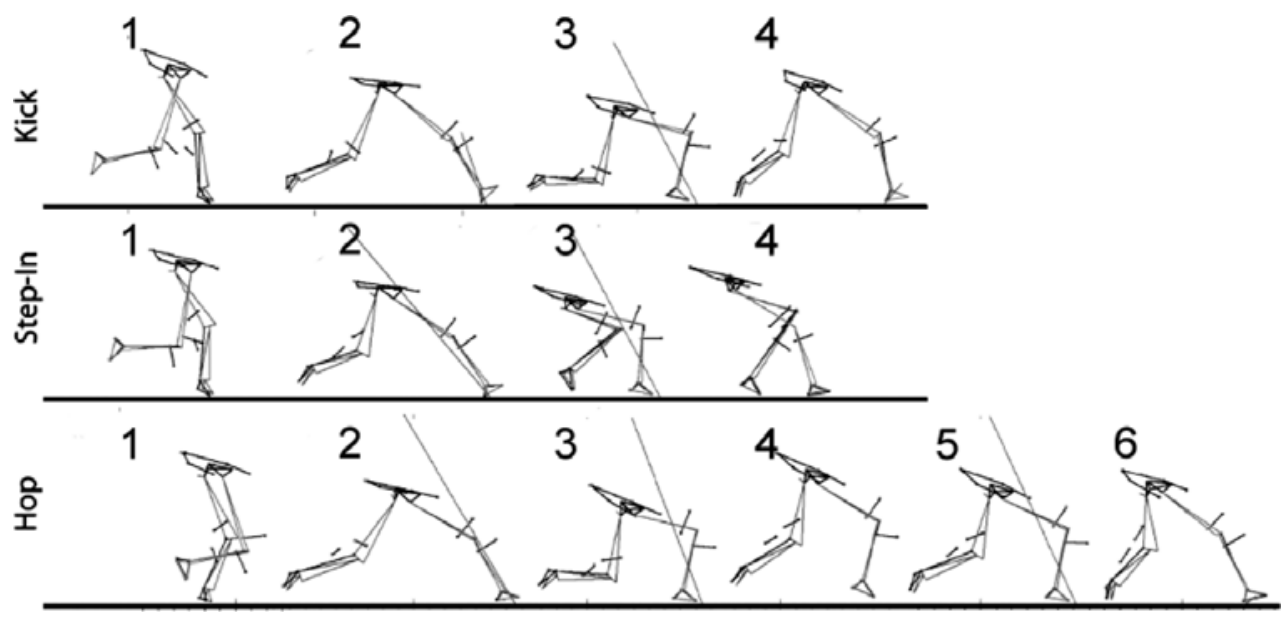

Figure 1. Visualization of the lower limb during the three lunge tasks. The stick figure is comprised of the hip, the dominant and non-dominant limbs respectively. Images taken from CODAmotion software. The kick lunge uses predominantly the dominant limb for accepting the weight of the body during the braking phase (2 to 3 ) and changing movement direction at lunge recovery (3 to 4), where limb dominance is identified by the racket hand. In the step-in lunge, the non-dominant limb is pulled towards the dominant limb at recovery (3 to 4) to assist in raising the body from peak flexion. The hop lunge incorporates a hop during the weight acceptance phase (3 to 5), before lunge recovery (5 to 6). 


\section{Biomechanical investigation}

\section{Participants}

With the approval of the local ethics committee, nine male first- and second-team players from the student badminton squad (age $20 \pm 2.12$ years; height $1.79 \pm 0.06 \mathrm{~m}$; weight $70.58 \pm$ $7.39 \mathrm{~kg}$ ) were recruited for the study. Each player performed three standardized lunging tasks on a simulated badminton court in the laboratory. The participants were all actively taking part in singles badminton competition (national level) and had at least six years experience in the sport. The data presented here are therefore representative of experienced male competitors. All participants signed an informed consent form and were informed of the general requirements of the experiment.

\section{Experimental design}

Upon signing the informed consent form, the participants were asked to perform a general 5min warm-up. After this warm-up, infrared markers for the recording of kinematics were attached to the participant using a number of lower limb wands supplied by Charnwood Dynamics Ltd. Thereafter, the participants performed a task familiarization routine consisting of a number of repeats of the three lunge methods identified above (Figure 1) within a simulated badminton court (Figure 2). The experiment was started once the participant and the investigator were satisfied with the performance of the lunge tasks. Participants were instructed to stand at the start position facing the net with their shoulders parallel to the net. From this position the participants moved along a wooden walkway and performed either a kick, hop or step-in lunge in response to a shuttle feed exercise and returned to the start position (see Figure 1). The lunge started with the non-dominant limb touching down at the start line (1), followed by extension of the dominant limb in front of the body making initial contact at impact (2), where limb dominance was determined from the racket hand. The same heel-first contact method was used in all lunges. The non-dominant limb remained extended to the back until maximal loading of the dominant limb (3). The drive-off phase (4) was performed using the dominant limb with a posteriorly extended non-dominant limb in the kick lunge, while the non-dominant limb was pulled towards the dominant limb in the step-in lunge to assist in the lunge recovery. The hop lunge used a brief hop (4) between the first and a second contact phase (5). At the second contact and drive-off phase (5 and 6), the nondominant limb was pulled medially to create a wide stance and assist in lunge recovery. 


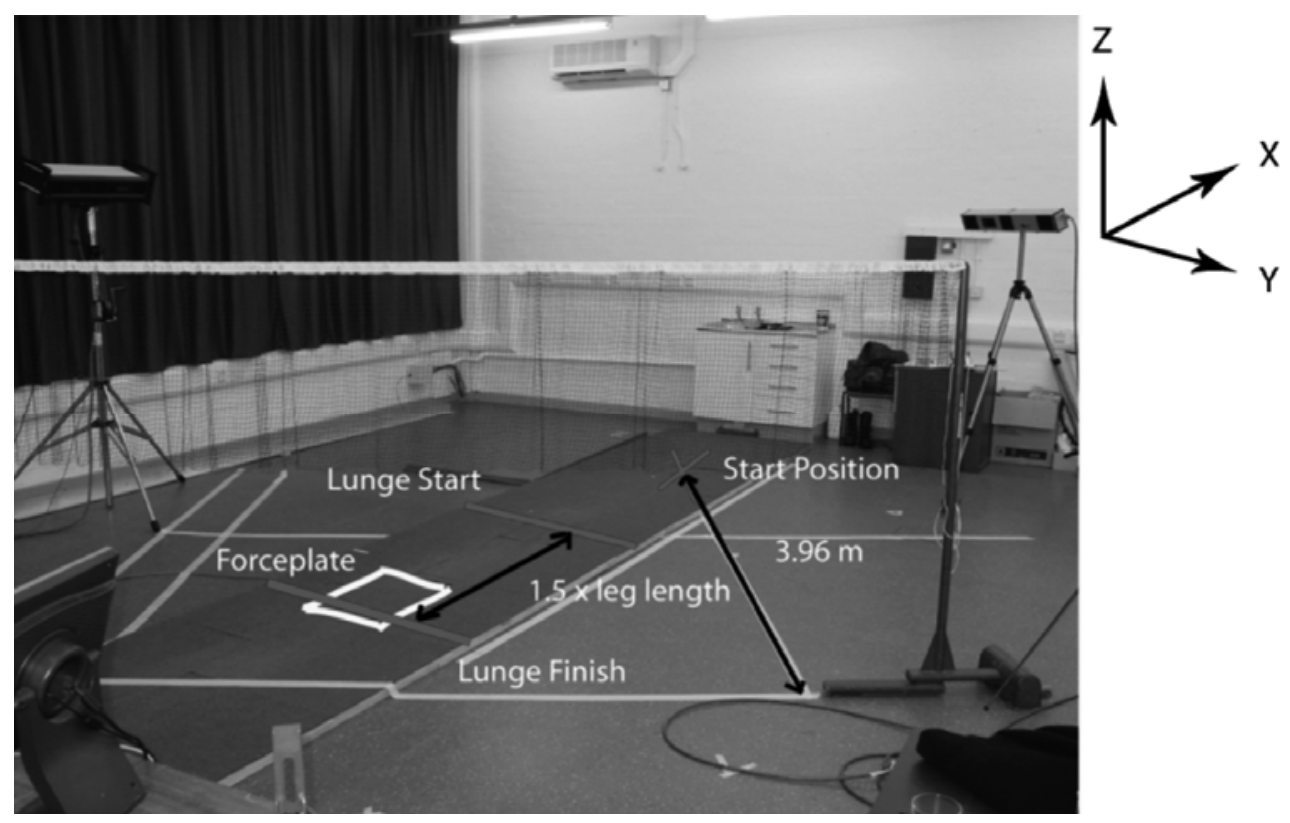

Figure 2. All tasks were performed on a simulated badminton court to aid the participant in understanding the requirements and ensure natural performance. Court tramlines (white) of a half court were accurately measured and marked out using masking tape. The start position was $3.96 \mathrm{~m}$ away from the net, along the central line of the court. Lunge start and finish lines were marked out using duct tape. The location of the lunge start was adjusted for each individual and was set at 1.5 times leg length. The location of the force plate is highlighted (rectangle). X, Y, and Z coordinates were defined with reference to the force-plate as indicated.

Participants were instructed to perform the task at maximum effort, while maintaining proper lunging form, to recreate a competitive environment. For the shuttle feed, the investigator manually threw the shuttle over the net with the shuttle landing in the front court area, from where the player returned the shuttle with the racket. Following preliminary testing, all lunge tasks were performed using a fixed lunge distance of 1.5 times leg length (vertical distance between the anterior superior iliac spine and the ground). This was deemed to be the largest distance that allowed for successful recovery using all three lunge methods. Lunge start and finish points were marked out as visual reference for the participant during familiarization and the investigator during data gathering to determine successful completion of each lunge task. Each participant performed seven successful repeats of the three lunge tasks, where a successful task repeat consisted of correct foot placement at the lunge start line, contact of the dominant limb with the centre of the force-plate, contact with the shuttlecock, and recovery to the starting position. Task order was randomized to mitigate order effects.

\section{Materials}

A Kistler (Type 9286A, Kistler, Switzerland) mobile multi-component force plate, integrated into the walkway, was used to record ground reaction forces at $200 \mathrm{~Hz}$. Lower-limb kinematic data were recorded at $200 \mathrm{~Hz}$ using a two-camera Cartesian Optoelectronic Dynamic Anthropometer (CODA, Charnwood Dynamics Ltd., UK) and a wand system for infrared marker attachment supplied by Charnwood Dynamics. Three-dimensional marker 
using gait analysis software by Charnwood Dynamics (CODAmotion V6.68). Kinematic and kinetic data were exported to Microsoft Excel for further analysis.

\section{Analysis}

Stance phase was defined as the period of time from initial contact to final lift-off from the force-plate by the dominant limb (i.e. containing both contact phases and the brief aerial phase of the hop lunge). Contact and lift-off were determined from the vertical reaction force (cut-off magnitude $=15 \mathrm{~N}$ ). Ground reaction force was analysed for seven task repeats per participant. For some individual repeats, the marker data sets were incomplete due to technical limitations of the motion capture equipment. Therefore, data averages of five trials per participant were chosen for the analysis of the kinetic data.

Approach speed and task durations (total time, stance phase, and recovery phase durations) were determined from kinematic and force data. Total task duration was defined as the time taken for the posterior superior iliac spine (PSIS) marker to travel horizontally from a predefined location ( $2.25 \mathrm{~m}$ from the far edge of the force-plate) to the force-plate and return to this location. Stance phase duration was derived from the force data, while recovery duration was the time taken by the PSIS marker to travel to the predefined location from final contact with the force-plate.

\section{Statistics}

All data were found to have normal distribution following skewness and kurtosis tests outlined by Vincent (2005). Statistical analysis of the influence of lunge method on performance variables (approach speed and task duration) and biomechanical variables (ground reaction force, joint moments and powers) was performed using repeated-measures analysis of variance (ANOVA) in SPSS. Paired samples $t$-tests were performed when appropriate to identify the contributors to a significant trend. Statistical significance was set at $P<0.05$.

\section{Results}

\section{Speed and durations}

Speed and duration data are summarized in Table II. There was no significant difference in mean approach speed $\left(F_{2,16}=0.679, P=0.513\right)$ or mean total task duration $\left(F_{2,16}=2.802\right.$, $P=0.09$ ) between the lunge methods despite a trend towards a slightly longer total task duration of the hop lunge. Duration of the stance phase $\left(F_{2,16}=39.819, P<0.001\right)$ and recovery phase $\left(F_{2,16}=6.467, P<0.01\right)$ was significantly affected by lunge method. Mean 
step-in $(P<0.001)$ lunges, with no significant difference between the latter two $(P=0.385)$. The recovery phase was significantly shorter for the hop than step-in lunge $(P<0.01)$, with no significant effects on recovery duration between the kick and hop $(P=0.122)$ or kick and step-in $(P=0.144)$ lunge methods.

\section{Table II. Summary of the group means for speeds and durations}

\section{Kick Step-in Hop}

$\begin{array}{llll}\text { Approach speed }\left(\mathrm{m} \cdot \mathrm{s}^{-1}\right) & 2.69 \pm 0.29 & 2.74 \pm 0.35 & 2.65 \pm 0.32 \\ \text { Total duration (s) } & 2.1 \pm 0.13 & 2.14 \pm 0.13 & 2.2 \pm 0.12 \\ \text { Stance phase duration (s) } & 0.62 \pm 0.06 & 0.63 \pm 0.07 & 0.78 \pm 0.03 \\ \text { Recovery duration (s) } & 0.87 \pm 0.1 & 0.91 \pm 0.12 & 0.81 \pm 0.1\end{array}$

\section{Ground reaction force}

Mean ground reaction force (GRF) curves for the kick, hop, and step-in lunge tasks are presented in Figure 3 for a representative participant. Following the classification by Lees and Hurley (1994), five phases can be clearly identified in all lunge scenarios:

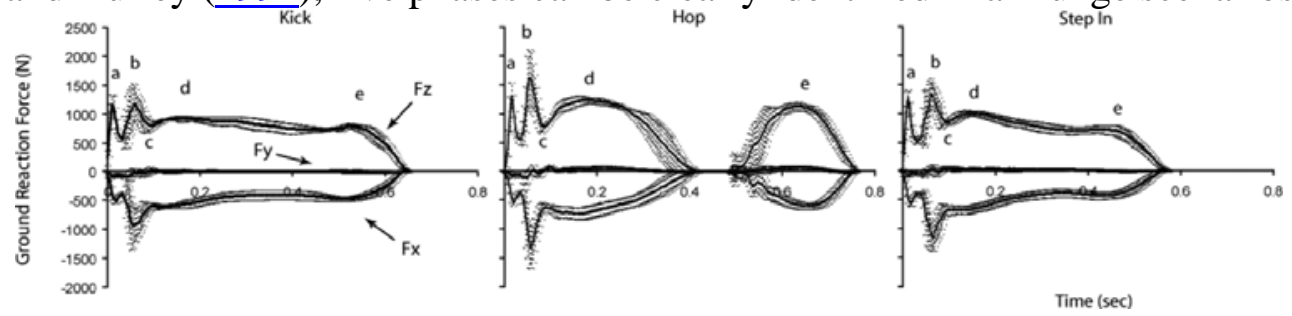

Figure 3. Vertical (Fz), horizontal (Fx), and transverse (Fy) ground reaction force (GRF) in three badminton lunge tasks. Data represent the mean GRF for one participant performing kick, hop, and step-in lunge methods. Five force phases were identified (a, b, c, d, e). Ground reaction force is presented in Newtons $(\mathrm{N})$. Error bars are used to visualize the magnitude of data variability between trials within the individual. The horizontal (Fx) and vertical (Fz) GRF curves display the most dominant force response, while little force is produced in the transverse plane.
a. initial impact peak (heel strike transient);
b. secondary impact peak (impact loading);
c. amortization;
d. weight acceptance (loading); and
e. drive-off.

Statistical analysis of the relationship of GRF phase and lunge method (summarized in Figure 4) was performed for horizontal and vertical force values. A significant effect of lunge method on mean peak vertical GRF was observed for the loading $\left(F_{2,16}=9.246, P<0.01\right)$ and drive-off $\left(F_{2,16}=85.47, P<0.001\right)$ phases. Pair-wise comparison indicated that the hop lunge resulted in significantly larger peak forces compared with the kick (loading: $P<0.01$; drive-off: $P<0.001$ ) and step-in (loading: $P<0.01$; drive-off: $P<0.001$ ) lunges, with no significant differences between the kick and step-in lunges (loading: $P=0.353$; drive-off: $P=0.418$ ). There was a small but significant effect of lunge method on phase (c) 
$\left(F_{2,16}=3.951, P<0.05\right)$, but no significant differences were observed in any of the pairwise tests (kick vs. hop: $P=0.057$; kick vs. step-in: $P=0.598$; hop vs. step-in: $P=0.054$ ).

Furthermore, no significant effect of lunge method was observed for phase (a) $\left(F_{2,16}=0.284\right.$, $P=0.757)$ or phase (b) $\left(F_{2,16}=2.208, P=0.142\right)$.
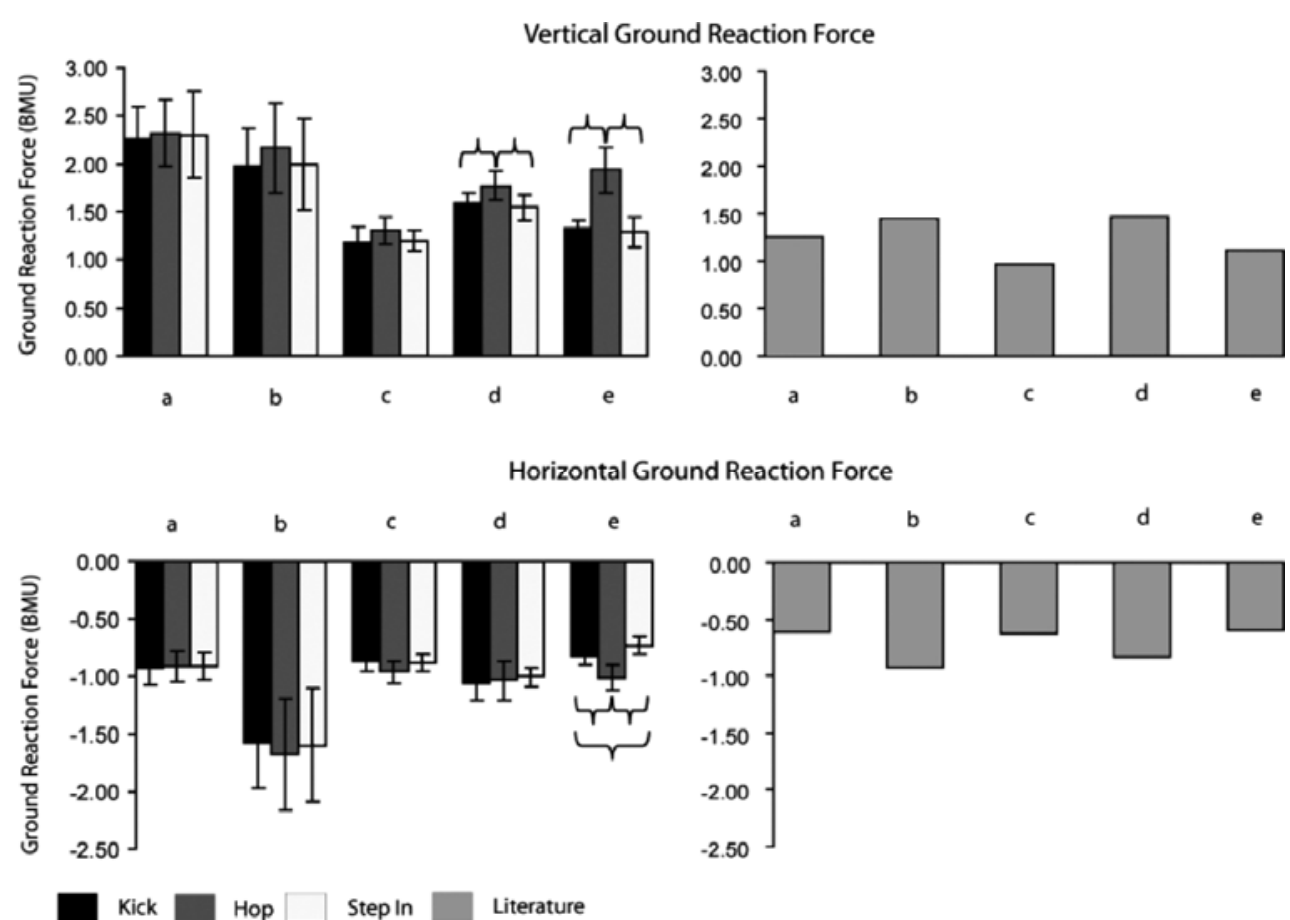

Figure 4. Summary of the findings of the relationship between ground reaction force and lunge method. Braces indicate significant differences between lunge pairs. The findings from this investigation were generally larger than those quoted in the literature for a typical badminton lunge (Lees and Hurley 1994).

Lunge method had a significant effect on horizontal forces at drive-off $\left(F_{2,16}=50.693\right.$, $P<0.001$ ). Peak force during the hop lunge was significantly larger than during the kick $(P<0.001)$ or step-in $(P<0.001)$ lunges, and significantly lower forces were produced by the step-in than the kick lunge $(P<0.01)$. No significant effects were observed for mean force peaks during phase (a) $\left(F_{2,16}=0.231, P=0.797\right)$, phase (b) $\left(F_{2,16}=0.654, P=0.533\right)$ or phase (d) $\left(F_{2,16}=0.438, P=0.653\right)$. Again, a small difference was observed for phase (c) $\left(F_{2,16}=3.719, P<0.05\right)$, although the pairwise comparisons showed no significant effect of lunge method (kick vs. hop: $P=0.057$; kick vs. step-in: $P=0.522$; hop vs. step-in: $P=0.061)$.

\section{Joint moments}

The mean peak hip, knee, and ankle moments were analysed for the kick (MK), the first and second peak moment of the hop (MH1 and MH2 respectively), and the step-in lunge (MS) (see Figure 5). The results of the statistical analysis (summarized in Figure 6) indicated no significant effect of lunge method on peak extensor moments at the hip $\left(F_{3,24}=0.133\right.$, $P=0.94)$ or knee joint $\left(F_{3,24}=2.436, P=0.089\right)$. Lunge method had a significant effect at the ankle joint $\left(F_{3,24}=4.171, P<0.05\right)$, where the ankle plantar flexor moment at MH2 of the 
lunge. No other significant relationships were observed at the ankle joint (MK vs. MH1: $P=0.307$; MK vs. MS: $P=0.839$; MH1 vs. MH2: $P=0.149$; MH1 vs. MS: $P=0.353$ ).
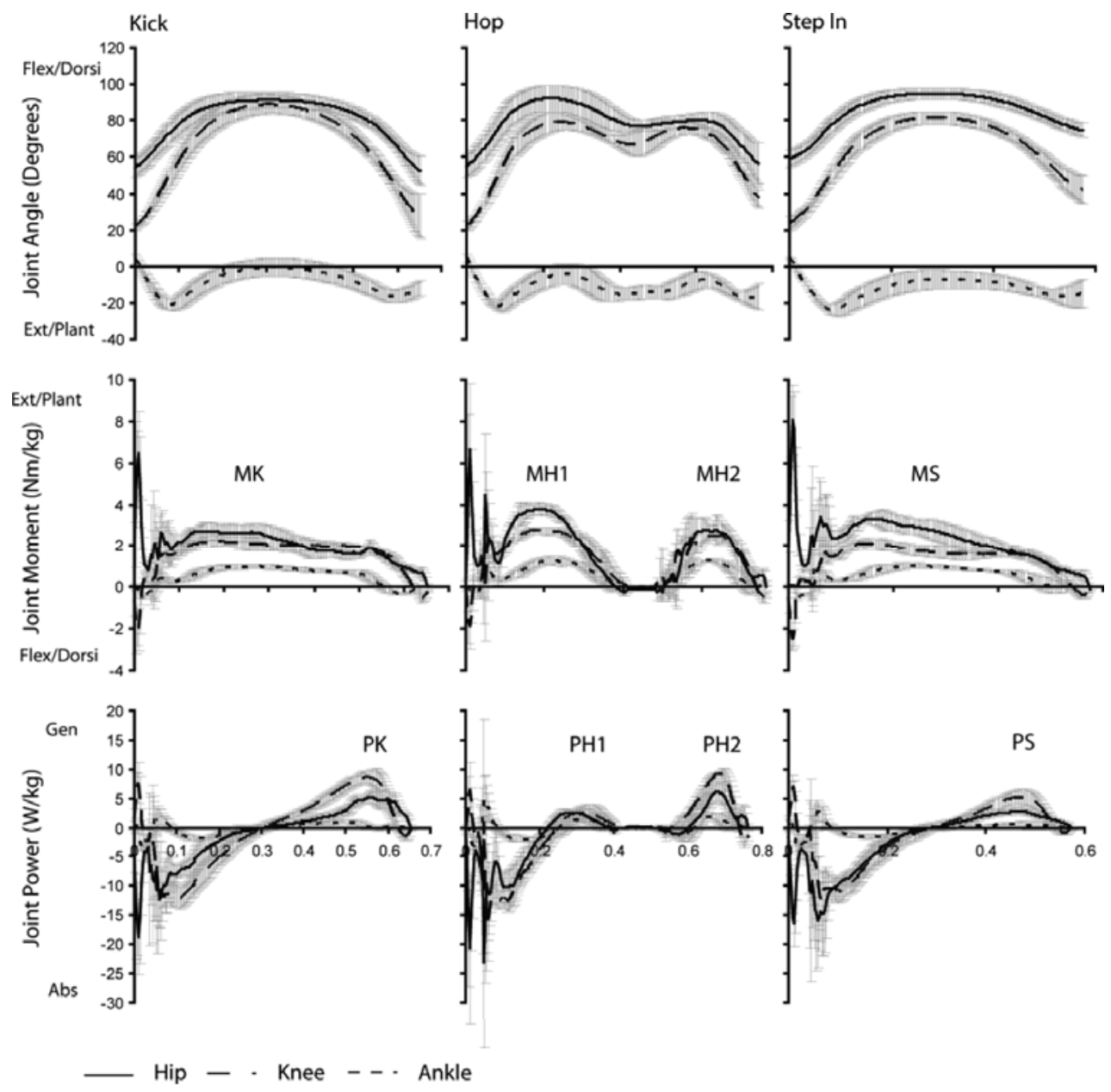

Figure 5. Summary of the mean joint angles, moments, and powers for one participant performing the kick, hop, and step-in lunges. Positive angles represent hip and knee flexion and ankle plantar flexion. Positive moments represent hip and knee extensor and ankle plantar flexor moments, and positive joint power values indicate periods of power generation. Important joint moment and power phases of the kick (MK; PK), hop (MH1, MH2; PH1, PH2), and step-in (MS; PS) lunges are indicated. Error bars indicate the data range between trials for the individual.

\section{Joint powers}

The mean peak hip, knee, and ankle joint powers were analysed for the kick (PK), the first and second peak moment of the hop (PH1 and PH2 respectively), and the step-in lunge (PS) (see Figure 5). The findings of the statistical analysis are presented in Figure 7. A significant effect of lunge method on mean peak joint power was observed at the hip joint $\left(F_{3,24}=16.141, P<0.001\right)$. We found that PK was significantly larger than PH1 $(P<0.01)$ and PS $(P<.05)$, with smaller values for PH1 than PS $(P<0.01)$. Furthermore, PH2 was found to be significantly larger than PH1 $(P<0.001)$ and PS $(P<0.05)$ but not significantly different from PK $(P=0.573)$. Peak knee power was also significantly affected by lunge 
$(P<0.01)$, PS $(P=0.001)$, and PH1 $(P<0.001)$. Furthermore, $\mathrm{PH} 1$ was smaller than PK $(P<0.001)$ and PS $(P=0.001)$, while there was no significant difference between PK and PS $(P=0.253)$. Finally, lunge method also affected mean peak ankle power $\left(F_{3,24}=9.44\right.$, $P<0.001)$. Here, PH2 was significantly larger than PK $(P<0.01)$, PS $(P<0.01)$, and PH1 $(P<0.05)$. However, there were no significant differences between $\mathrm{PK}$ and PS $(P=0.462)$, PH1 and PS $(P=0.469)$ or PH1 and PK $(P=0.085)$.

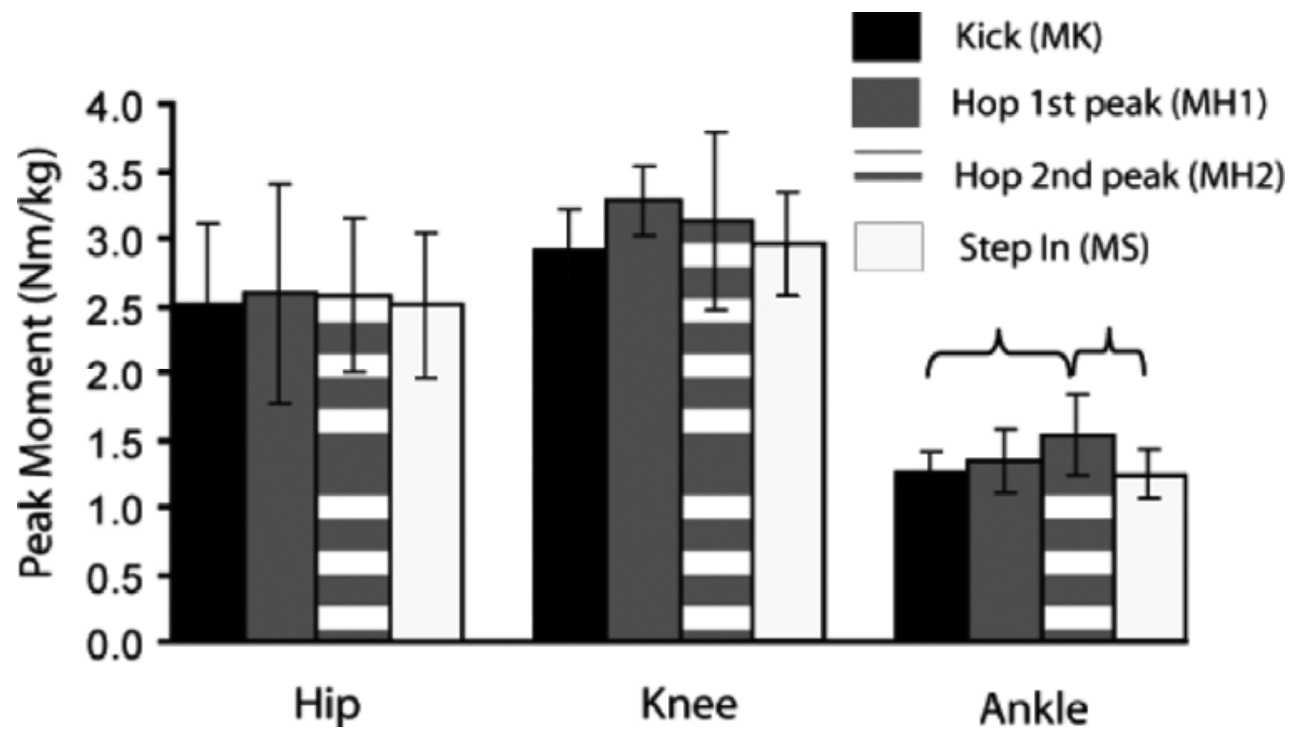

Figure 6. Summary of the findings for the peak hip, knee, and ankle moments produced during the kick, hop, and step-in lunges. Braces indicate significant differences between lunge pairs.

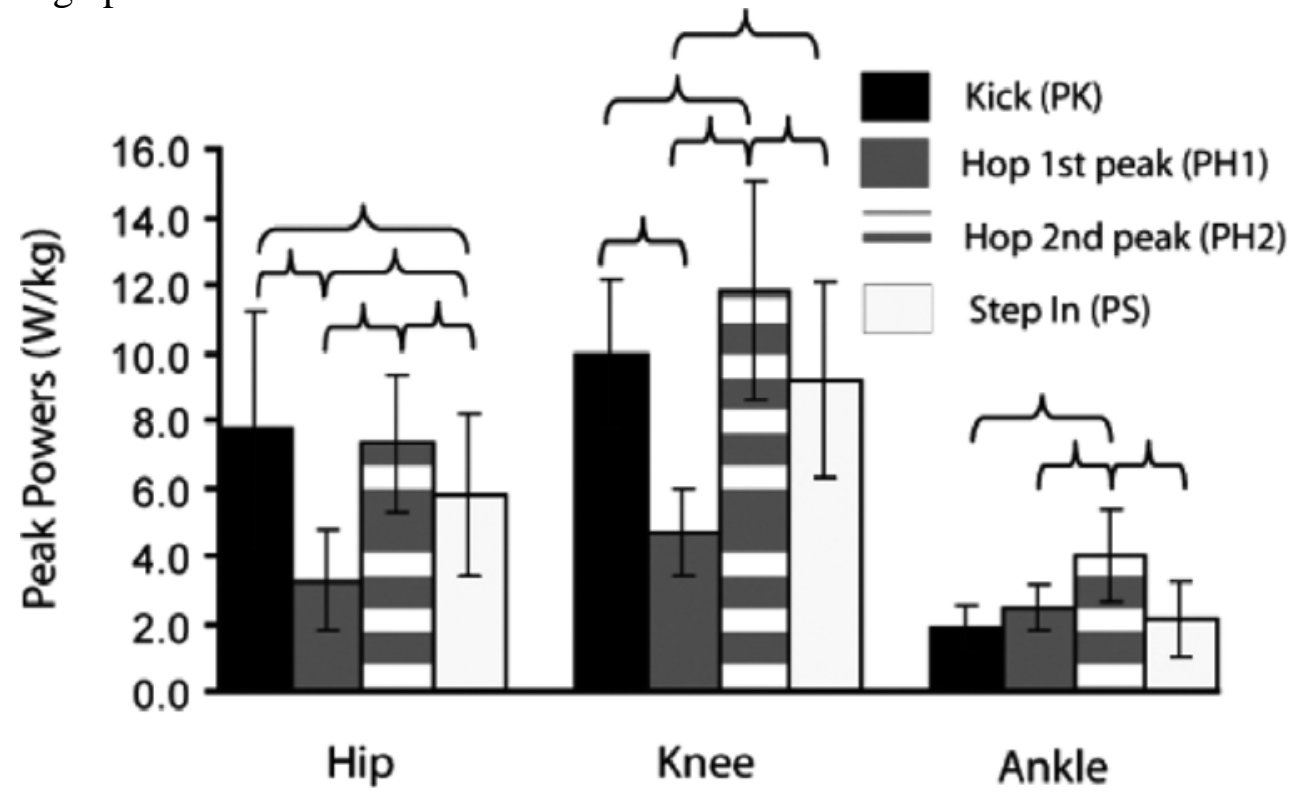

Figure 7. Summary of the findings for peak hip, knee, and ankle power produced during the kick, hop, and step-in lunges. Braces denote significant differences in peak power between lunge pairs.

\section{Discussion}


A video-based pilot study identified a lunge frequency of $~ 15 \%$ in competitive singles

badminton. It was the objective of the biomechanical investigation to assess differences in joint mechanics and performance criteria between three badminton-specific lunge tasks. These data are essential to gain specific information on the biomechanical demands of different lunge tasks, the potential for reduced joint loading, as well as enhancing task performance.

Mean peak ground reaction forces identified in this study were generally in excess of those quoted by Lees and Hurley (1994) for typical forces in a badminton lunge movement performed by experienced players (see Figure 4). The discrepancy in peak forces may be due to a difference in movement magnitude between the studies or an underestimation of peak force due to the lower sampling frequency employed in their study $(100 \mathrm{~Hz})$. However, no details of lunge distance or timings for lunge task completion were available. Nevertheless, very similar dynamic trends can be observed in the data between studies (Figure 4), indicating that despite the differences in magnitude, lunge movements appeared to be dynamically similar at the level of ground reaction forces. There were no significant differences in the early impact forces (heel-strike transient, impact loading, and amortization) between the lunge methods tested, despite low $P$-values in the hop/kick and hop/step-in comparisons at amortization. This is likely due to initial contact with the ground being made using the same heel strike method by all participants in all lunge scenarios (see Figure 1). However, as shown in Figure 4, peak vertical force during loading was larger for the hop lunge, which is likely due to the generation of extensor force by the knee joint to lift the leg off the ground for the hop phase prior to the secondary ground contact. Furthermore, during the secondary contact phase, the participants were able to apply larger vertical and horizontal forces to the ground at drive-off. These findings are in line with observations by Fukashiro and colleagues (Fukashiro, Komi, Jarvinen, \& Miyashita, 1995) for hopping and partly explain the faster recovery phase of the hop lunge, since the application of larger horizontal and vertical forces at the secondary ground contact of the hop lunge would allow the participants to generate more speed at drive-off and therefore recover more quickly. Despite the involvement of the non-dominant limb in the step-in lunge, aiding the dominant limb at drive-off, there was no significant reduction of vertical force at drive-off compared with the kick lunge. However, the significant reduction in horizontal force indicates a shift in the action of the dominant limb, maintaining an important function in raising the body from the flexed position, but contributing to a lesser extent to horizontal push-off and the return to the base position.

To further assess the functional significance of the contribution of the joints of the dominant limb to the stance phase of the three lunge methods, it is necessary to examine the moments and powers at the joints. There was no significant difference in peak joint moments at the hip or knee joints. However, at the ankle the second contact phase of the hop lunge was significantly larger than for either the kick or step-in lunge. The cause of this increase in ankle moment is likely due to the use of the forefoot at second ground contact, which allowed for a larger contribution of the ankle joint through ankle plantar flexion. This idea is substantiated by the finding of significantly larger ankle positive power (Figure 7) in the second contact phase of the hop lunge compared with the kick and step-in lunges. These 
findings are consistent with the current view of the effect of the muscular stretch-shortening cycle (Bobbert \& van Ingen Schenau, 1990; Cavagna, 1978; Edman, Elzinga, \& Noble, 1978; Jacobs, Bobbert, \& van Ingen Schenau, 1993; Jaric, Gavrilovic, \& Ivancevic, 1985) and the utilization of elastic energy (van Ingen Schenau, Bobbert, \& de Haan, 1997a) on the increase of power generation by the musculature. By using the hop lunge, the task is effectively transformed into a hop movement in the second part of the lunge. The tendons - the Achilles tendon in particular - are recognized for their important function in returning stored elastic energy (Biewener, 1997; Josephson, 1999; van Ingen Schenau, Bobbert, \& de Haan, 1997b; Zatsiorsky 1997). Data reported by Fukashiro et al. (1995) show that the contribution of elastic energy to the mechanical work done at the Achilles tendon is larger during hopping exercises than squat jump or countermovement jump. Furthermore, Ker and colleagues (Ker, Bennett, Bibby, Kester, \& Alexander, 1987) showed that not only the Achilles tendon but also the foot return energy by stretching and recoil (35\% and 17\% respectively during running). In addition, the stretching of the muscle itself causes a potentiation of the contractile machinery, which increases with the speed of stretch, and therefore enhances the ability of the muscle to do work (van Ingen Schenau et al. 1997a). By changing the orientation of the foot during stance in the hop lunge, it is therefore possible to enhance force production by taking advantage of elastic energy stored in the foot and Achilles tendon as well as enhancing the contractile function of the gastrocnemius and soleus.

Similarly, at the knee joint the secondary contact phase of the hop lunge enabled the quadriceps muscles to generate significantly more positive power than either the kick or stepin lunges (see Figure 7). As for the ankle joint, it is thought that this increase in power is likely related to an enhanced stretch-shortening mechanism and therefore indicates enhanced mechanical efficiency. Furthermore, mean positive joint powers for the first contact phase of the hop lunge were significantly smaller than for any other lunge method, indicating reduced concentric contraction, which may be beneficial for reducing muscular fatigue.

At the hip, peak extensor power during the kick and second contact phase of the hop lunge were significantly larger than that produced during the step-in. This finding supports the idea that the step-in lunge reduces muscular work during recovery and may be beneficial for the reduction of muscular fatigue. As for the knee joint powers, mean peak hip joint power was lower for the primary contact phase of the hop lunge than the kick or step-in lunges, which may be beneficial for reducing muscular fatigue.

Despite significantly longer stance duration, total task duration was only slightly and nonsignificantly longer for the hop lunge. This indicates that, at the lunge magnitude used, different methods appeared to have a minor influence on task performance. The quicker recovery phase of the hop lunge compensated for the longer stance phase, and the faster recovery may indeed be advantageous in a situation where it is necessary to carry the momentum on beyond the start position to respond to, for example, a clearing shot played to the back of the court. Using the hop lunge instead of the kick or step-in lunges might therefore result in the player arriving at the shuttle faster when continued movement beyond the start position is necessary.

Despite the efforts made to recreate a competitive environment, our results are a reflection of the demands of lunging in a controlled laboratory setting. Lunge performance is affected by differences in technique, individual preference, and parameters that include body mass, leg length, and flexibility (Cronin, McNair, \& Marshall, 2001, 2003). Therefore, only skilled male badminton players with ample experience were selected to represent a consistent sample 
for the assessment of lunge technique. However, an effect of variations in technique and physical differences cannot be discounted as contributors to data variation between participants. Since the hop lunge represented a novel technique to most participants, the required adaptation to the technique was kept to a minimum, which reduced the technical demands and was perceived as an easy adjustment by all participants. Furthermore, it appears likely that, within the dynamic setting of a badminton competition, selection of lunge type is affected to a great extent by lunge distance and player location on court before and at the time of playing a shot (i.e. movement direction). A net shot following a back-court clear by the opponent, for example, may require a large lunge and adoption of a step-in recovery. Nevertheless, establishing the demands within a comparable lunge magnitude provides a vital baseline for comparison, and further investigation of the extreme cases of lunge performance would be beneficial to further clarify movement demands in the sport.

\section{Conclusion}

Our results provide evidence for the reduction of muscular demands during recovery when adopting a step-in lunge technique, which may be beneficial for reducing muscle fatigue and soreness. Furthermore, it is evident that the hop lunge increases the force output of the dominant limb by optimizing force-generating processes, which may prove advantageous for quick recovery and movement beyond the start position and adoption of this movement strategy may be recommended to enhance the contribution of the knee and ankle joint to the mechanism of lunge recovery. The data therefore provide evidence in favour of modifying established movement routines to enhance the performance of the athlete. It should be the focus of future studies to establish the effect of further modifications of the lunge routine, adjusting the weight acceptance phase, to identify possibilities for reducing eccentric muscular contraction and thereby reducing muscular fatigue and injury risk potential.

\section{References}

- 1. Adrian, M. and Klinger, A. (1976) A biomechanical analysis of the fencing lunge. Medicine and Science in Sports and Exercise 8 , p. 56.

- 2. Badminton Association of England (2005) Level 1: Assistant coach training manual BAE , Milton Keynes

- 3. Biewener, A. A. (1997) Effects of elastic energy storage on muscle work and efficiency. Journal of Applied Biomechanics 13 , pp. 422-426.

- 4. Bobbert, M. F. and van Ingen Schenau, G.J. (1990) Isokinetic plantar flexion: Experimental results and model calculations. Journal of Biomechanics 23 , pp. 105119.

- $\quad$ 5. Cavagna, G. A. Asmussen, E. and Jørgensen, K. (eds) (1978) Aspects of efficiency and inefficiency of terrestrial locomotion. Biomechanics VI-A pp. 3-26. University Park Press , Baltimore, MD

- $\quad$ 6. Cronin, J. B., McNair, P. J. and Marshall, R. N. (2001) Relationship between strength qualities and motor skills associated with court performance. Journal of Human Movement Studies 40 , pp. 207-224.

- 7. Cronin, J. B., McNair, P. J. and Marshall, R. N. (2003) Lunge performance and its 
determinants. Journal of Sports Sciences $\mathbf{2 1}$, pp. 49-57. [informaworld]

- 8. Edman, K. A., Elzinga, G. and Noble, M. I. (1978) Enhancement of mechanical performance by stretch during tetanic contractions of vertebrate skeletal muscle fibres. Journal of Physiology 281 , pp. 139-155.

- 9. Fukashiro, S., Komi, P. V., Jarvinen, M. and Miyashita, M. (1995) Achilles tendon loading during jumping in humans. European Journal of Applied Physiology and Occupational Physiology 71 , pp. 453-458.

- $\quad$ 10. Gebhardt, D. L. (1981) Force and power production in the fencing lunge. Journal of Biomechanics 14 , p. 494.

- 11. Heijne, A., Fleming, B. C., Renstrom, P. A., Peura, G. D., Beynnon, B. D. and Werner, S. (2004) Strain on the anterior cruciate ligament during closed kinetic chain exercises. Medicine and Science in Sports and Exercise 36 , pp. 935-941.

- 12. Jacobs, R., Bobbert, M. F. and van Ingen Schenau, G.J. (1993) Function of monoand biarticular muscles in running. Medicine and Science in Sports and Exercise 25 , pp. 1163-1173.

- $\quad$ 13. Jaric, S., Gavrilovic, P. and Ivancevic, V. (1985) Effects of previous muscle contractions on cyclic movement dynamics. European Journal of Applied Physiology and Occupational Physiology 54 , pp. 216-221.

- 14. Josephson, R. K. (1999) Dissecting muscle power output. Journal of Experimental Biology 202 , pp. 3369-3375.

- $\quad$ 15. Ker, R. F., Bennett, M. B., Bibby, S. R., Kester, R. C. and Alexander, R. M. (1987) The spring in the arch of the human foot. Nature 325 , pp. 147-149.

- 16. Lees, A. and Hurley, C. Reilly, T., Hughes, M. and Lees, A. (eds) (1994) Forces in a badminton lunge movement. Science and racket sports pp. 249-256. E \& FN Spon , London

- 17. Stuart, M. J., Meglan, D. A., Lutz, G. E., Growney, E. S. and An, K. N. (1996) Comparison of intersegmental tibiofemoral joint forces and muscle activity during various closed kinetic chain exercises. American Journal of Sports Medicine 24 , pp. 792-799.

- $\quad$ 18. Thijs, Y., van Tiggelen, D., Willems, T., De Clercq, D. and Witvrouw, E. (2007) Relationship between hip strength and forward plane posture of the knee during a forward lunge. British Journal of Sports Medicine 41 , pp. 723-727.

- 19. van Ingen Schenau, G.J., Bobbert, M. F. and de Haan, A. (1997a) Does elastic energy enhance work and efficiency in the stretch-shortening cycle?. Journal of Applied Biomechanics 13 , pp. 389-415.

- 20. van Ingen Schenau, G.J., Bobbert, M. F. and de Haan, A. (1997b) Mechanics and energetics of the stretch-shortening cycle: A stimulating discussion. Journal of Applied Biomechanics 13 , pp. 484-496.

- 21. Vincent, W. J. (2005) Statistics in kinesiology Human Kinetics , Champaign, IL

- 22. Zatsiorsky, V. M. (1997) The review is nice: I disagree with it. Journal of Applied Biomechanics 13 , pp. 479-483.

\section{List of Figures}




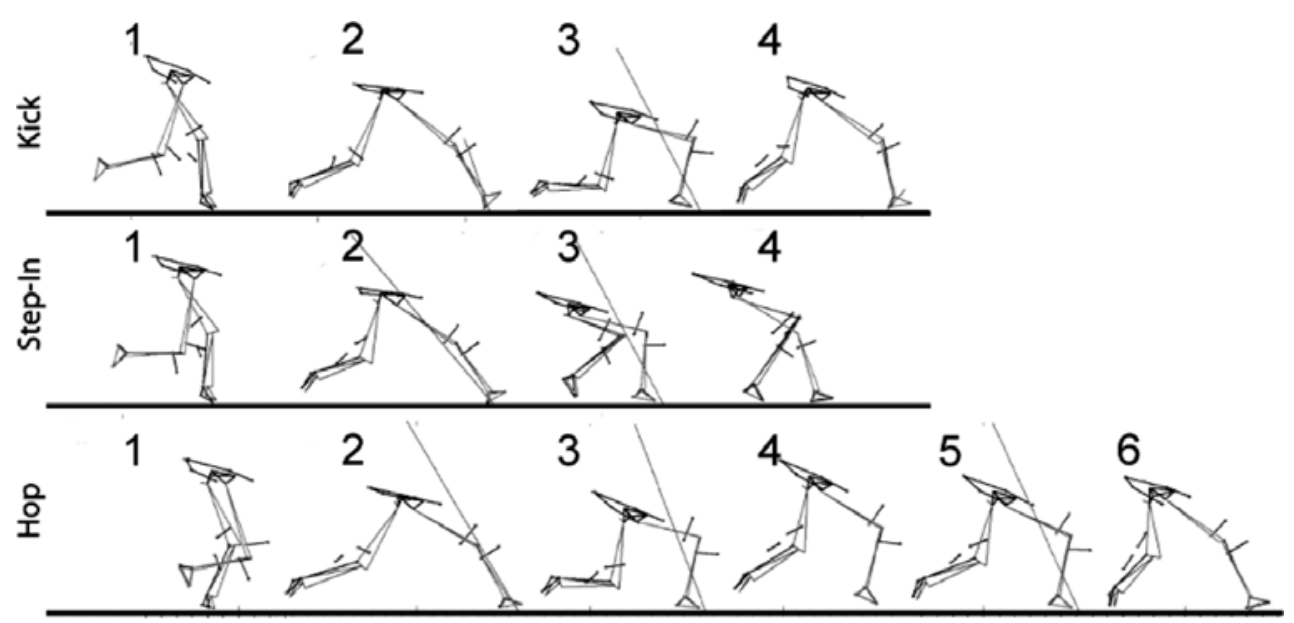

Figure 1. Visualization of the lower limb during the three lunge tasks. The stick figure is comprised of the hip, the dominant and non-dominant limbs respectively. Images taken from CODAmotion software. The kick lunge uses predominantly the dominant limb for accepting the weight of the body during the braking phase (2 to 3 ) and changing movement direction at lunge recovery (3 to 4), where limb dominance is identified by the racket hand. In the step-in lunge, the non-dominant limb is pulled towards the dominant limb at recovery (3 to 4) to assist in raising the body from peak flexion. The hop lunge incorporates a hop during the weight acceptance phase (3 to 5), before lunge recovery (5 to 6).

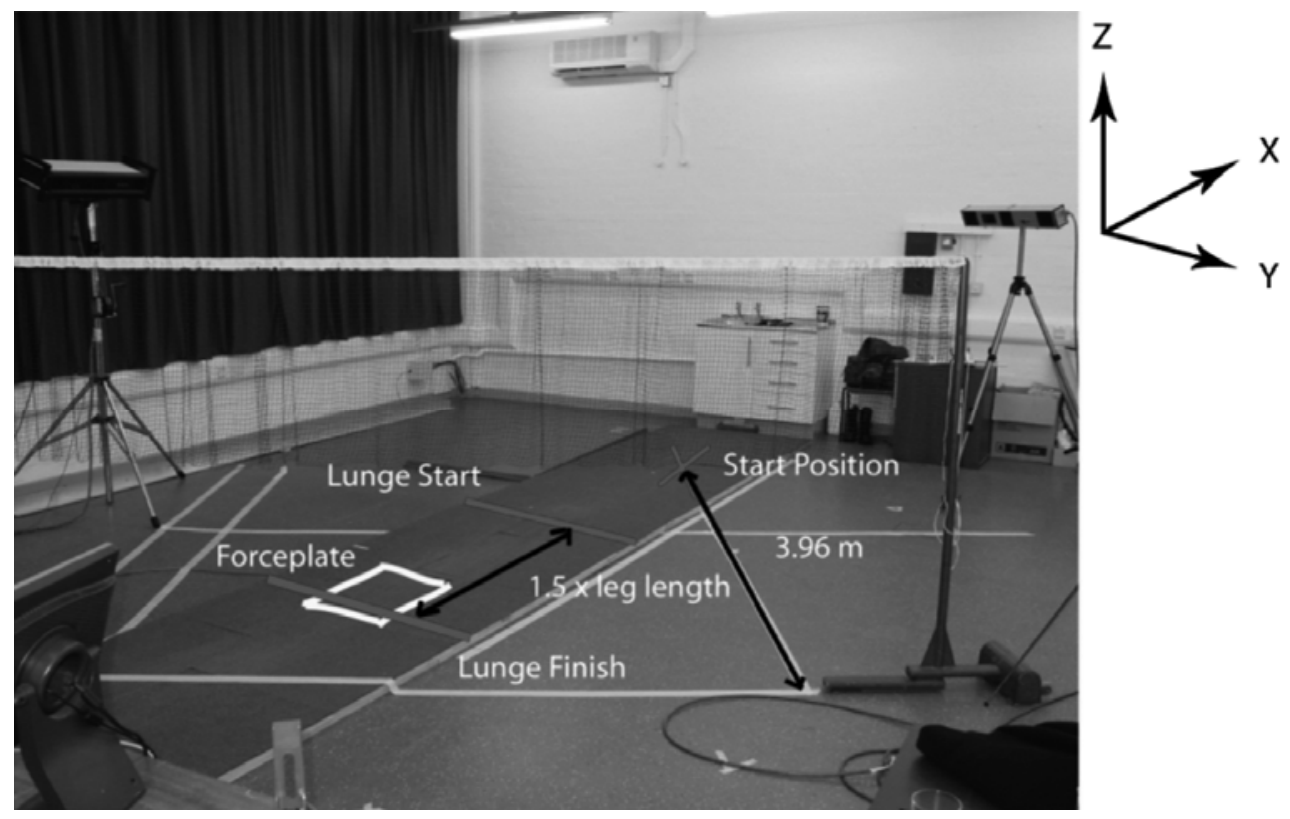

Figure 2. All tasks were performed on a simulated badminton court to aid the participant in understanding the requirements and ensure natural performance. Court tramlines (white) of a half court were accurately measured and marked out using masking tape. The start position was $3.96 \mathrm{~m}$ away from the net, along the central line of the court. Lunge start and finish lines were marked out using duct tape. The location of the lunge start was adjusted for each individual and was set at 1.5 times leg length. The location of the force plate is highlighted (rectangle). X, Y, and Z coordinates were defined with reference to the force-plate as indicated. 

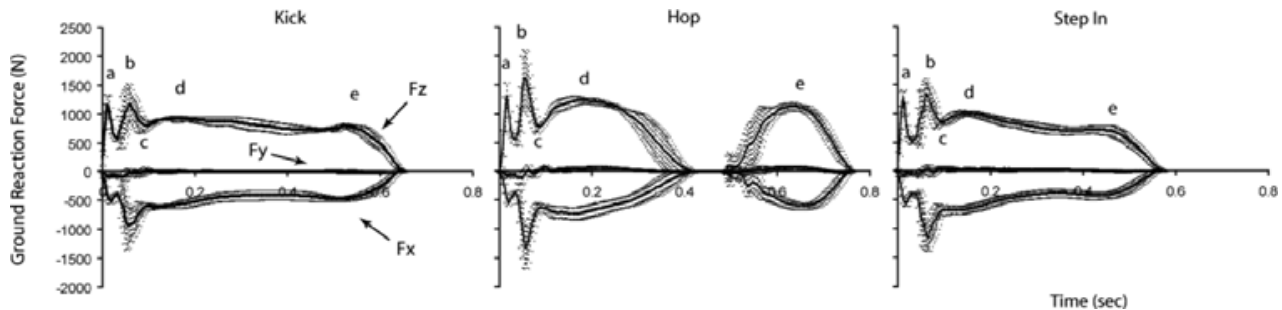

Figure 3. Vertical (Fz), horizontal (Fx), and transverse (Fy) ground reaction force (GRF) in three badminton lunge tasks. Data represent the mean GRF for one participant performing kick, hop, and step-in lunge methods. Five force phases were identified (a, b, c, d, e). Ground reaction force is presented in Newtons $(\mathrm{N})$. Error bars are used to visualize the magnitude of data variability between trials within the individual. The horizontal (Fx) and vertical (Fz) GRF curves display the most dominant force response, while little force is produced in the transverse plane.
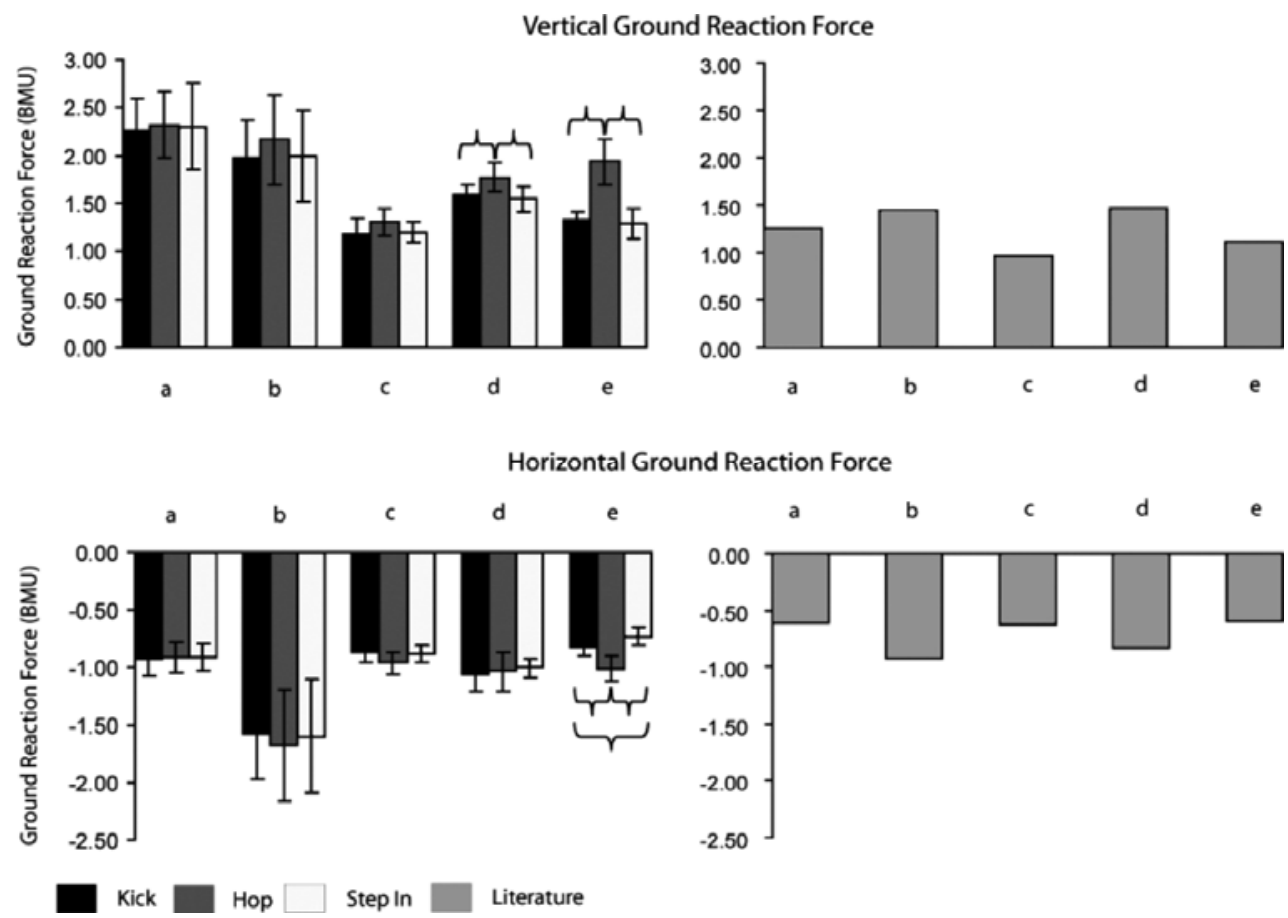

Figure 4. Summary of the findings of the relationship between ground reaction force and lunge method. Braces indicate significant differences between lunge pairs. The findings from this investigation were generally larger than those quoted in the literature for a typical badminton lunge (Lees and Hurley 1994). 

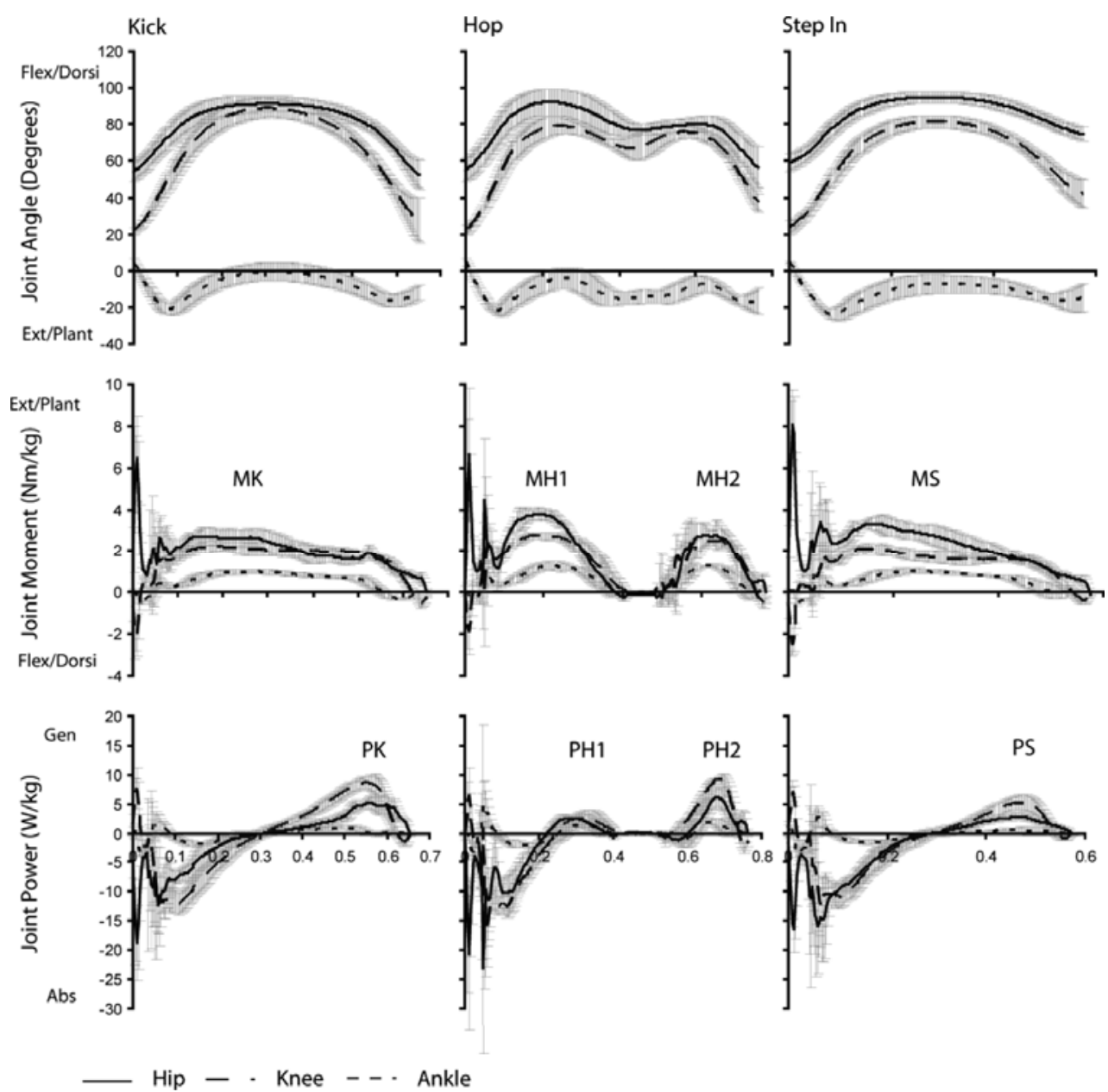

Figure 5. Summary of the mean joint angles, moments, and powers for one participant performing the kick, hop, and step-in lunges. Positive angles represent hip and knee flexion and ankle plantar flexion. Positive moments represent hip and knee extensor and ankle plantar flexor moments, and positive joint power values indicate periods of power generation. Important joint moment and power phases of the kick (MK; PK), hop (MH1, MH2; PH1, PH2), and step-in (MS; PS) lunges are indicated. Error bars indicate the data range between trials for the individual.

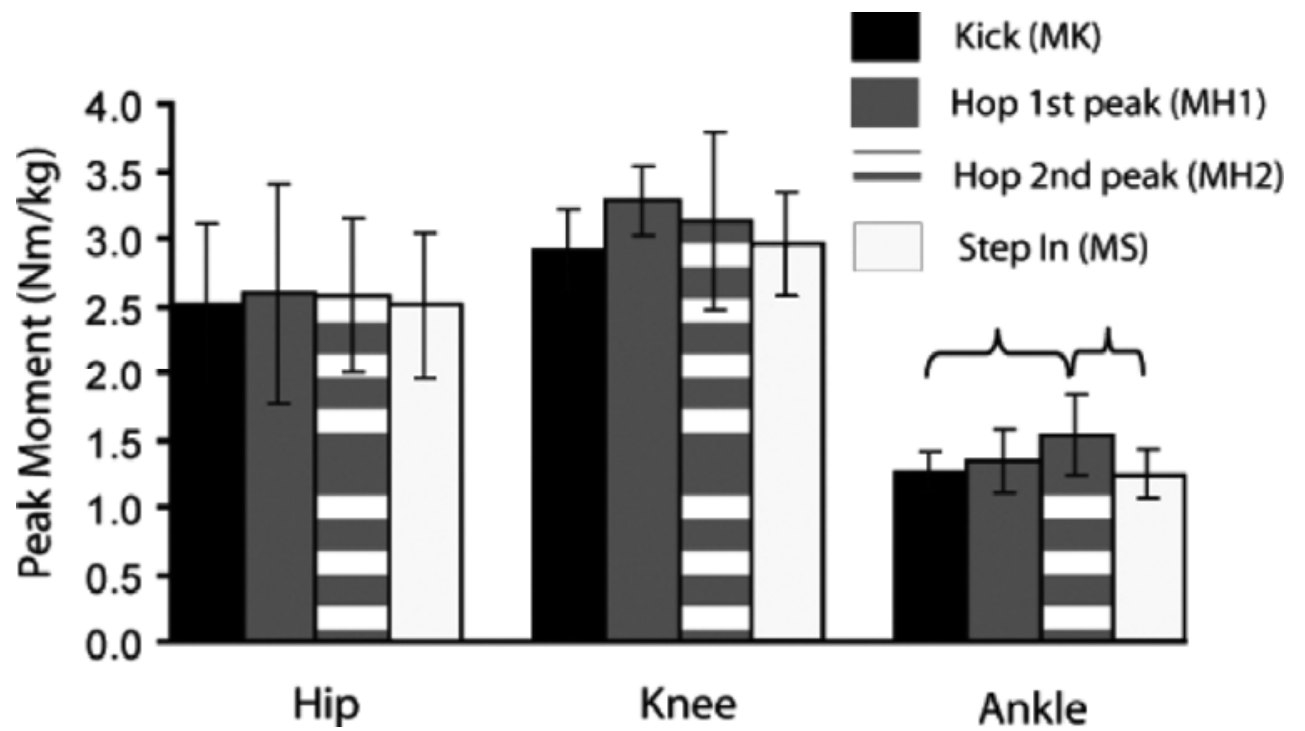

Figure 6. Summary of the findings for the peak hip, knee, and ankle moments produced 
during the kick, hop, and step-in lunges. Braces indicate significant differences between lunge pairs.

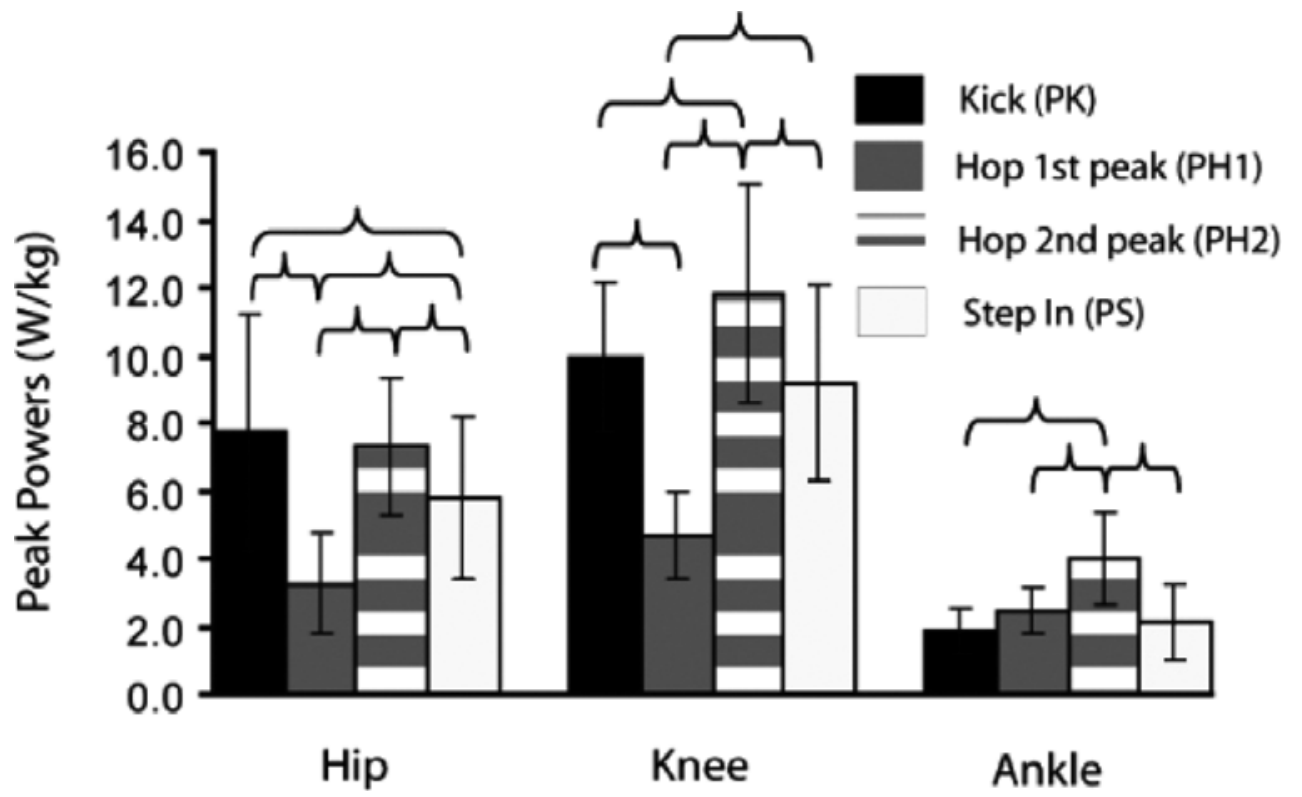

Figure 7. Summary of the findings for peak hip, knee, and ankle power produced during the kick, hop, and step-in lunges. Braces denote significant differences in peak power between lunge pairs.

\section{List of Tables}

Table I. Summary of participant and video information

\begin{tabular}{|c|c|c|}
\hline Games analysed & $\begin{array}{l}\text { Number of } \\
\text { players }\end{array}$ & $\begin{array}{c}\text { Mean } \\
\text { sampling } \\
\text { duration (s) }\end{array}$ \\
\hline
\end{tabular}

BUSA $=$ British Universities Sports Association.

Men, $\quad 2 \times$ World Cup semi-final, $2 \times$ World Cup

international quarter-final, $1 \times$ Thomas Cup Final, 10

10729155

Women,

international

$1 \times$ Asian Games Final

Men, national $3 \times$ BUSA finals, $3 \times$ Satellite

Tournament

Women,

national

$5 \times$ BUSA finals

722192

8

58951

Table II. Summary of the group means for speeds and durations

\section{Kick Step-in Hop}
Approach speed $\left(\mathrm{m} \cdot \mathrm{s}^{-1}\right)$
$2.69 \pm 0.29 \quad 2.74 \pm 0.35 \quad 2.65 \pm 0.32$
Total duration (s)
$2.1 \pm 0.13 \quad 2.14 \pm 0.13 \quad 2.2 \pm 0.12$ 
Stance phase duration (s) $\quad 0.62 \pm 0.06 \quad 0.63 \pm 0.07 \quad 0.78 \pm 0.03$

Recovery duration (s)

$0.87 \pm 0.1 \quad 0.91 \pm 0.12 \quad 0.81 \pm 0.1$ 